\title{
PEEK interbody devices for multilevel anterior cervical discectomy and fusion: association with more than 6-fold higher rates of pseudarthrosis compared to structural allograft
}

\author{
Zoe E. Teton, BS, ${ }^{1}$ Barry Cheaney II, BS, James T. Obayashi, BS, ${ }^{1}$ and Khoi D. Than, MD ${ }^{1,2}$ \\ ${ }^{1}$ Department of Neurological Surgery, Oregon Health \& Science University, Portland, Oregon; and 2Department of Neurosurgery, \\ Duke University, Durham, North Carolina
}

OBJECTIVE Common interbody graft options for anterior cervical discectomy and fusion (ACDF) include allograft and polyetheretherketone (PEEK). PEEK has gained popularity due to its radiolucent properties and a modulus of elasticity similar to that of bone. PEEK devices also result in higher billing costs than allograft, which may drive selection. A previous study found a 5-fold higher rate of pseudarthrosis with the use of PEEK devices compared with structural allograft in single-level ACDF. Here the authors report on the occurrence of pseudarthrosis with PEEK devices versus structural allograft in patients who underwent multilevel ACDF.

METHODS The authors retrospectively reviewed 81 consecutive patients who underwent a multilevel ACDF and had radiographic follow-up for at least 1 year. Data were collected on age, sex, BMI, tobacco use, pseudarthrosis, and rate of reoperation for pseudarthrosis. Logistic regression was used for data analysis.

RESULTS Of 81 patients, 35 had PEEK implants and 46 had structural allograft. There were no significant differences between age, sex, smoking status, or BMI in the 2 groups. There were 26/35 (74\%) patients with PEEK implants who demonstrated radiographic evidence of pseudarthrosis, compared with $5 / 46(11 \%)$ patients with structural allograft $(p<$ 0.001 , OR 22.2). Five patients (14\%) with PEEK implants required reoperation for pseudarthrosis, compared with 0 patients with allograft $(p=0.013)$.

CONCLUSIONS This study reinforces previous findings on 1-level ACDF outcomes and suggests that the use of PEEK in multilevel ACDF results in statistically significantly higher rates of radiographic pseudarthrosis and need for revision surgery than allograft. Surgeons should consider these findings when determining graft options, and reimbursement policies should reflect these discrepancies.

https://thejns.org/doi/abs/10.3171/2019.11.SPINE19788

KEYWORDS anterior cervical discectomy and fusion; ACDF; pseudarthrosis; polyetheretherketone; PEEK; allograft; cervical

$\Lambda$ NTERIOR cervical discectomy and fusion (ACDF) is considered by many surgeons as the gold standard for treating cervical spondylosis with myelopathy, radiculopathy, disc herniation, and other cervical diseases refractory to conservative management. ${ }^{1,6}$ Success with these procedures is largely determined by the ability to provide decompression of neural structures, reduce potential instability, preserve disc height and maintain align- ment, and ultimately achieve bony fusion using the appropriate interbody graft. .,11 $^{-11}$

As this surgical technique has continued to improve, the options for graft material have increased. Autograft, often obtained from the anterior iliac crest, was originally used in $\mathrm{ACDF}$ procedures and preferred given its lack of immunogenicity and minimal disease transmission. ${ }^{17,25}$ However, the harvesting of the iliac crest bone comes with add-

ABBREVIATIONS ACDF = anterior cervical discectomy and fusion; $\mathrm{DBM}=$ demineralized bone matrix; rhBMP = bone morphogenetic protein

SUBMITTED July 10, 2019. ACCEPTED November 14, 2019.

INCLUDE WHEN CITING Published online January 24, 2020; DOI: 10.3171/2019.11.SPINE19788. 
ed morbidity, including donor site pain, lateral cutaneous nerve palsy, and structural weakness, as well as increased operative time, blood loss, and rate of infection., ${ }^{1,6,11,17,19}$ To avoid these complications, allograft substitutes, including cortical, cancellous, and composite cadaver bone, are now frequently used. However, these grafts do come with the theoretical risk of transmission of infectious diseases such as hepatitis, HIV, and tuberculosis. ${ }^{1,3}$

In an effort to avoid the morbidity of autograft and the possible disease transmission of allograft, synthetic interbody fusion devices were developed. The device material can be titanium, ceramic, carbon fiber, or polyetheretherketone (PEEK), with PEEK cages being the most common. ${ }^{1,26}$ PEEK is broadly accepted due to its nonresorbable and radiolucent properties, as well as having a modulus of elasticity similar to that of bone. ${ }^{5,8}$ The use of synthetic cages results in increased billing costs per surgical level compared with allograft, which may drive graft selection. ${ }^{21} \mathrm{~A}$ previous study at our institution retrospectively examined the incidence of radiographic pseudarthrosis in patients who underwent a 1-level ACDF with either a PEEK or structural allograft implant, ${ }^{7}$ and found a 5-fold higher rate of pseudarthrosis with the use of PEEK devices as opposed to structural allograft in single-level ACDF. Here we report on the occurrence of pseudarthrosis of PEEK devices versus structural allograft in patients who underwent multilevel ACDF. This is the first study, to our knowledge, to compare pseudarthrosis and reoperation rates in a large cohort of patients who underwent multiple-level ACDF.

\section{Methods}

In this retrospective, single-center study, all consecutive ACDF procedures from July 2011 to November 2017 were reviewed. Thirteen different attending surgeons (10 neurological surgeons and 3 orthopedic surgeons) performed the operative procedures. Patients undergoing a single-level ACDF and patients undergoing front-back procedures were not included. Patients who did not have at least 1 year of follow-up with either a cervical spine radiograph, flexion and extension radiograph, or CT were also excluded. Implant selection, duration of follow-up, and acquisition of follow-up imaging were performed according to the practice pattern of the individual surgeon. The study was approved by the institutional review board, with a waiver of consent.

Electronic medical records were reviewed for demographic data, smoking status, medical comorbidities, procedure indication, type of graft material utilized, and radiographic evidence of pseudarthrosis. The presence of pseudarthrosis was defined as the lack of solid bony growth across the disc space or facets after at least 1 year of radiographic follow-up on either static radiographs or CT scans, or the presence of motion on cervical flexion and extension radiographs. The most recent available imaging for every patient was reviewed. If a patient had images obtained with multiple modalities, greater emphasis was placed on the CT scan or flexion and extension radiographs. All postoperative imaging was reviewed by a radiologist (which was part of the standard of care, and thus the radiologist was blinded to the study) and 3 of the authors (K.D.T., Z.E.T., and B.C.). Records were further reviewed by the researchers for any additional surgical intervention that was warranted beyond the index surgery. All records were also reviewed for occurrence of postoperative infection.

In general, the surgical technique was as follows. $\mathrm{Pa}-$ tients were positioned supine on the operating room table after receiving general anesthetic. The neck was prepped and draped in a sterile fashion. An anterior dissection to the ventral spine was performed, and retractors were placed. The discectomies were performed. The interbody grafts were placed. If a plate was used, it was affixed and fastened in place using screws. If the grafts had incorporated instrumentation (i.e., zero-profile devices), those shims or screws were affixed without a plate. The wound was then closed in layers.

\section{Statistical Analysis}

Statistical analysis was undertaken using SPSS Statistics version 24 (IBM Corp.), and p values were considered significant at $<0.05$. Logistic regression analysis was used to determine whether there were statistically significant correlations between the rate of pseudarthrosis and graft materials (PEEK vs allograft). A Pearson's correlation test was used to determine if there was a significant correlation between graft material and smoking status in the study patients. A Spearman's rho test was used to determine if there was a statistically significant level of correlation between reoperation rates and graft material used (PEEK vs allograft), as well as between smoking status and pseudarthrosis in both PEEK and allograft groups. Spearman's rho was also used to determine if there was a correlation between graft material placed in PEEK devices and rate of pseudarthrosis and reoperation. In patients who received PEEK implants, Pearson's correlation test was used to determine if there was a statistically significant difference in pseudarthrosis rates in standalone versus plated implants, and Spearman's rho test was used to determine the same for reoperation rates. Lastly, a Student t-test was used to assess for differences between radiographic follow-up times and BMI values between the 2 groups (PEEK vs allograft). Spearman's rho was used, instead of Pearson's correlation, to test for statistical significance of correlations between 2 variables when cross-tabulation of the reviewed variables yielded cell counts of less than 5 .

\section{Results}

In total, 294 patients underwent multilevel ACDF during the 6-year and 4-month data collection period, 81 (28\%) of whom met the inclusion criteria. Of these 81 patients, 35 (43\%) patients had PEEK implants and $46(57 \%)$ had structural allograft implants (Table 1). The majority of these surgeries were 2-level procedures $(61 / 81 ; 75 \%)$, while $17(20 \%)$ procedures involved 3 levels, and $3(5 \%)$ involved 4 levels (Table 1). There were no significant differences between pseudarthrosis rates in patients who underwent a 2-level fusion $(25 / 61,41 \%)$ and those who underwent fusions of 3 or more levels $(6 / 20,30 \%)(p=$ 0.477). 
TABLE 1. Patient demographics

\begin{tabular}{lccc}
\hline & Allograft & PEEK & Total \\
\hline No. of patients & 46 & 35 & 81 \\
\hline No. of levels & & & \\
\hline 2 & 32 & 29 & 61 \\
\hline 3 & 12 & 5 & 17 \\
\hline 4 & 1 & 2 & 3 \\
\hline Mean age, yrs & 54 & 53 & $54^{*}$ \\
\hline Sex, $\mathrm{n}$ & & & \\
$\quad$ Male & 17 & 13 & 30 \\
\hline$\quad$ Female & 29 & 22 & 51 \\
\hline Smokers, $\mathrm{n}(\%)$ & $11(24)$ & $9(26)$ & $20(25)$ \\
\hline Mean BMl $\pm \mathrm{SD}, \mathrm{kg} / \mathrm{m}^{2}$ & $31.0 \pm 7.6$ & $31.7 \pm 7.8$ & $31.35 \pm 7.7^{*}$ \\
\hline
\end{tabular}

There was no statistical significance in any category.

${ }^{*}$ Mean value for total patient population.

In the PEEK group, the mean age at the time of surgery was 53.3 years (range 35-77 years), and in the allograft group it was 53.8 years (range $32-78$ years). There were 13 male and 22 female patients in the PEEK group, and 17 males and 29 females in the allograft group. There was no significant difference in BMI $(\mathrm{p}=0.673)$ or smoking status ( $p=0.804)$ between the PEEK and allograft groups, and every procedure across both groups was performed for degenerative changes. Three patients who received allografts were taking steroids at the time of their operation (prednisone or dexamethasone), but this use of immunosuppressive medications did not appear to affect healing as none of these patients exhibited pseudarthrosis at their most recent imaging follow-up. None of the patients who received PEEK were taking steroids.

As determined from the most recent imaging obtained, 37 (46\%) patients had cervical spine radiographs, 24 (29\%) had flexion and extension radiographs, and 20 (24\%) had cervical spine CT scans. The most recent postACDF follow-up imaging was obtained at the 12-month time point in $35 \%(16 / 46)$ of allograft patients and 9\% (3/35) of PEEK patients, at 12-18 months in 22\% (10/46) of allograft patients and 9\% (3/35) of PEEK patients, and at $18-24$ months in $4 \%(2 / 46)$ of allograft patients and $11 \%$ $(4 / 35)$ of PEEK patients. For the rest of the patients in each cohort, 39\% (18/46) of allograft patients and 71\% (25/35) of PEEK patients, the most recent imaging was obtained some time beyond the 24-month time point. Average radiographic follow-up was longer in the PEEK group (34 \pm 19.2 months) than in the structural allograft group (26.0 \pm 17.8 months, $\mathrm{p}=0.057$ ).

Of the 35 patients with PEEK implants, 26 (74\%) displayed radiographic evidence of pseudarthrosis (Fig. 1) compared with 5 of the 47 (11\%) with structural allograft ( $\mathrm{p}<0.001$, OR 22.2, 95\% CI 6.68-73.93; Table 2). Five patients (14\%) with PEEK implants required a revision operation for pseudarthrosis compared with 0 patients in the structural allograft group $(\mathrm{p}=0.013)$. Clinical indications for revision surgeries in the PEEK group included neck pain (2/5), myelopathy (2/5), and a combination of radiculopathy, myelopathy, and kyphosis $(1 / 5)$. The aver-
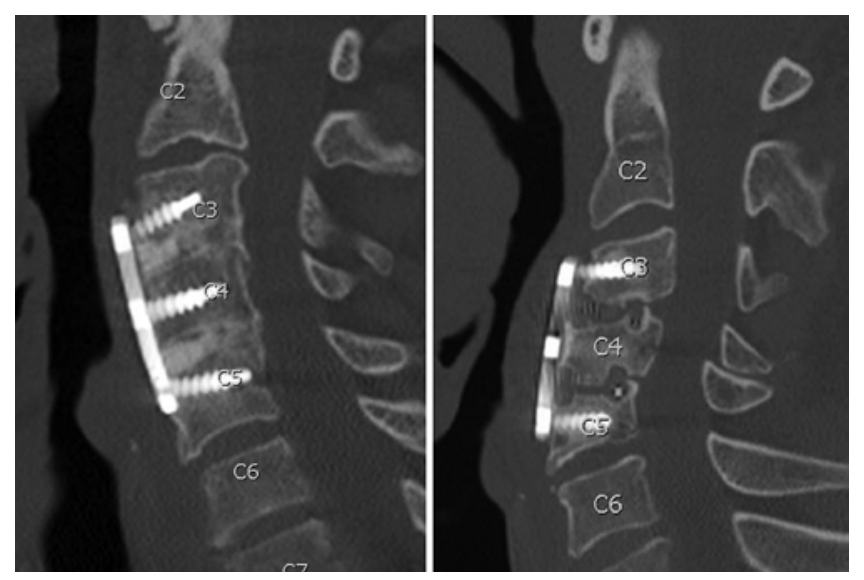

FIG. 1. Radiographs of ACDF patients. Left: Patient with a structural allograft implant healed 16 months after surgery. Right: Patient with a PEEK interbody graft and pseudarthrosis 35 months after surgery.

age interval between initial operation and diagnosis of pseudarthrosis was 12 months (range 7-18 months). The type of redo surgery was most commonly a posterior instrumented fusion (3/5), with 1 redo $\operatorname{ACDF}(1 / 5)$ and 1 redo ACDF combined with a new $\mathrm{ACDF}$ of an adjacent level $(1 / 5)$.

The average $\mathrm{T} 1$ slope was $24^{\circ}$ in the allograft group and $20^{\circ}$ in the PEEK group, while average cervical lordosis was $10^{\circ}$ in the allograft group and $7^{\circ}$ in the PEEK group. Neither of these variables was found to have a significant effect on fusion rates ( $\mathrm{T} 1$ slope, $\mathrm{p}=0.278$; lordosis, $\mathrm{p}=0.08$ ).

All PEEK cages were filled with either demineralized bone matrix (DBM) $(13 / 35,37 \%)$, a combination of DBM and local autograft $(10 / 35,29 \%)$, nonstructural allograft $(8 / 35,23 \%)$, a combination of DBM and allograft $(2 / 35$, $6 \%$ ), or local autograft $(1 / 35,3 \%)$. Fusion occurred with the use of nonstructural allograft $(3 / 8,37.5 \%), \mathrm{DBM}$ and autograft in combination $(3 / 10,30 \%)$, and DBM alone $(3 / 13,23 \%)$. Neither local autograft $(0 / 1)$ nor the combination of DBM and allograft (0/2) resulted in fusion. Reoperations occurred with the use of local autograft (1/1, $100 \%)$, the combination of DBM and allograft $(1 / 3,33 \%)$, DBM alone $(2 / 13,15 \%)$, and nonstructural allograft (1/8, $12.5 \%)$. The combined use of DBM and local autograft did not result in any reoperations (0/10). There was no statistically significant correlation between any particular PEEK graft choice and pseudarthrosis or reoperation rate, given the data available. It should be noted that there were insufficient data to determine if there was a correlation between

TABLE 2. Comparison of radiographic pseudarthrosis, need for revision surgery, and smoking status in patients with allografts versus PEEK implants

\begin{tabular}{lcrr}
\hline & Allograft, $n(\%)$ & PEEK, $n(\%)$ & $p$ Value \\
\hline Pseudarthrosis & $5(11)$ & $26(74)$ & $<0.001$ \\
\hline Revision surgery & $0(0)$ & $5(14)$ & 0.013 \\
\hline Smokers w/ pseudarthrosis & $1(20)$ & $6(23)$ & 0.463 \\
\hline
\end{tabular}


the use of local autograft as the PEEK graft choice and pseudarthrosis or reoperation rate (only 1 data point). The majority of PEEK cages were supplied by Stryker (25/35), while $9(9 / 35)$ were supplied by LDR Global, and one cage company was not specified. There was no significant difference in fusion rate based on the company that supplied the PEEK cage $(p=0.166)$. Structural allograft implants were made from cortical bone (6/46) materials or a combination of cortical and cancellous bone materials (34/46). In 6 cases, the allograft material was not specified in the patient documentation.

Postoperative wound infection occurred in 1 PEEK patient who eventually needed revision surgery for pseudarthrosis. The patient's initial surgery was complicated by a wound hematoma and a Propionibacterium infection that was treated with wound exploration, hematoma evacuation, and long-term amoxicillin administration. There were no reports of postoperative transmission of hepatitis or HIV in either group.

The majority of the PEEK implants were associated with plate and screw fixation $(22 / 35,63 \%)$, while the others involved the use of standalone devices $(13 / 35,37 \%)$. Radiographic evidence of pseudarthrosis was observed in $73 \%$ (16/22) of the PEEK implants with associated plates and $77 \%(10 / 13)$ of those without associated plates, which was not a significant difference $(\mathrm{p}=1.000)$. Revision surgeries were needed in 4 of the 5 patients with PEEK implants associated with plates, though this was not a significant correlation $(p=0.630)$ and was likely to have been related to the nearly 3 times as many plated PEEK implants than standalone devices. Thus, PEEK implants resulted in higher rates of pseudarthrosis and reoperations whether or not they were associated with plate and screw fixation, a finding that was also demonstrated in a reported study of single-level ACDF procedures at our institution. ${ }^{7}$ All structural allograft cases were associated with plate and screw fixation.

Smoking status was investigated to determine if there was a correlation between tobacco use and rates of pseudarthrosis in the 2 groups. Of those patients with radiographic evidence of pseudarthrosis, 20\% (1/5) in the allograft group and 23\% (6/26) in the PEEK group were current smokers (Table 1). There was no correlation between tobacco use and pseudarthrosis in either the PEEK $(\mathrm{p}=0.450)$ or allograft $(\mathrm{p}=0.142)$ groups.

Of the 13 surgeons who performed ACDF in this patient cohort, 3 used PEEK exclusively, 7 used allograft exclusively, and 3 used both PEEK and allograft. For the 3 surgeons who used both implant types, $100 \%$ of their cases of radiographic pseudarthrosis occurred in patients with PEEK implants $(54 \%, 7 / 13)$ compared with 0 cases with allograft use $(n=11)$. Secondary revision surgery was required for treatment in 1 of the 7 patients who developed pseudoarthrosis in this PEEK group.

\section{Discussion}

While other studies have compared rates of subsidence, dysphagia, and variation in the effects of bone morphogenetic protein (rhBMP) use between PEEK and allograft implants, this study is the first to our knowledge to ex- amine pseudarthrosis and reoperation rates exclusively in multiple-level ACDF procedures. ${ }^{10,20,26}$ Our results demonstrate a more than 6-fold increase in the rate of radiographic pseudarthrosis and a higher reoperation rate with the use of PEEK implants compared with structural allograft after at least 1 year of radiographic follow-up.

This retrospective study was conducted as a follow-up to a previous study on single-level ACDF procedures at our institution that demonstrated similar findings. ${ }^{7}$ In the prior study, 127 patients met inclusion criteria and were evaluated for rates of radiographic pseudarthrosis.7 Seventy-one of these patients received structural allograft, while 56 had PEEK implants, 82\% (46/56) of which were standalone devices. After at least 1 year of radiographic follow-up, radiographic pseudarthrosis was demonstrated in $51 \%(29 / 56)$ of patients with PEEK implants but in only $10 \%$ (7/71) of patients with structural allografts $(\mathrm{p}<0.001$, OR 9.82). Reoperation for pseudarthrosis was required for 7 patients $(12.5 \%)$ with PEEK implants compared with 1 patient $(1.4 \%)$ with structural allograft $(\mathrm{p}=0.01$, OR 10.00). Similarly, in the multiple-level ACDF cohort reported here, pseudarthrosis rates in patients who received PEEK implants were 6.7 times higher $(74 \%$ vs $11 \%$, p < 0.001 , OR 22.2) than in patients who received structural allografts, and the patients who received PEEK implants had a statistically significantly higher reoperation rate (14\% vs $0, p=0.013)$.

Despite initial excitement in the surgical community regarding PEEK as a suitable alternative in ACDF operations, the pitfalls to its use have become more apparent with time. A 2007 study comparing the use of PEEK and rhBMP-2 with allograft and demineralized bone matrix found no significant difference in clinical outcome at 2 years, but highlighted that the cost for patients who received implants with PEEK spacers was 3 times higher than that of the allograft implants. ${ }^{23}$ This study was followed by a 2015 systematic review that also showed fusion rates with PEEK to be comparable to those with allograft, but the authors noted that PEEK spacers demonstrated a lack of osteointegration and lamented the need for future studies with improved methodologies to reduce the risk of bias.

Pirkle et al. examined ACDF patients registered in the PearlDiver database from 2007 to 2016, and their study included over 4000 allograft patients and over 2000 PEEK patients. ${ }^{16}$ The authors found a nearly 3 -fold higher rate of nonunion in the PEEK group $(5.32 \%$ vs $1.97 \%, \mathrm{p}<0.01$ ) after 1 year. ${ }^{16}$ There was no statistically significant difference in the number of tobacco users or diabetes diagnoses between the 2 groups, and reoperation rates were not examined. Of course, it should be noted that the limitations of these databases are that they rely on billing codes exclusively, and individual radiographs are not examined. This increase in nonunion rates with PEEK compared with allograft was also demonstrated in work from Yson et al., who compared subsidence rates between PEEK and allograft implants used by one orthopedic surgeon. ${ }^{26}$ Their examination found no difference in subsidence rates between the 2 groups $(\mathrm{p}=0.89)$, but they did note that reoperation for pseudarthrosis was required in 4 of the patients in the PEEK group $(n=48)$ but in just 1 patient in 
the structural allograft group $(\mathrm{n}=19)$. Yson et al. did not assess these rate variances for significance, though the difference between the 2 groups of approximately $3 \%(8.3 \%$ PEEK vs 5.3\% allograft) is similar to that found by Pirkle et al.

This striking discrepancy in pseudarthrosis rates is of particular importance to spine surgeons for a number of reasons. First, pseudarthrosis is one of the most common complications in spine surgery and is detrimental when it leads to inappropriate segmental motion, as this can cause neck pain, radiculopathy, myelopathy, and instrument failure, and ultimately may require revision surgery. ${ }^{9}$ The risk of nonunion is especially prevalent in multiple-level procedures and has been reported to result in significantly higher Neck Disability Index (NDI) scores and lower rates of improvement in neck pain..$^{22}$ This increased likelihood of pseudarthrosis with the use of PEEK was reflected in the cohort we describe as rates of pseudarthrosis increased by $23 \%$ in the PEEK group and $1 \%$ in the allograft group compared with the rates in our previous study on singlelevel ACDF procedures.

Second, even asymptomatic patients with radiographic pseudarthrosis are at significant risk of developing symptoms over time, and this is especially true for patients who undergo multiple-level procedures. Phillips et al. published a retrospective study with a mean follow-up of 5.1 years in which they examined the long-term outcomes of patients diagnosed with pseudarthrosis following ACDF and found that two-thirds of those patients eventually became symptomatic. ${ }^{15}$ These findings were expanded upon in a review published by Lee et al. that examined the long-term prognosis of ACDF patients diagnosed with nonunion 1 year after surgery. ${ }^{9}$ The authors found that $28 \%$ of patients diagnosed with pseudarthrosis after 1 year had still not achieved bony fusion after 2 years. This rate jumped to $33 \%$ for only those patients who had 2-level operations, and to $40 \%$ for only those patients who had 3-level operations. While the authors suggested that pseudarthrosis cannot be definitively diagnosed until at least 2 years postoperatively, they acknowledged that fusion was less likely to occur spontaneously in patients who underwent multiple-level surgery and suggested that intervention should be considered before that time point, especially in patients who become symptomatic. This was the case in the cohort we describe as all 5 patients with symptomatic pseudarthrosis in the PEEK group were indicated for reoperation within 18 months.

Finally, the number of ACDF procedures performed is growing and the patient population receiving them is also increasing in age and complexity. From 2002 to 2009 , there was a $200 \%$ increase in cervical spine surgeries, which was specifically attributed to a striking increase in ACDF procedures. ${ }^{14}$ ACDFs accounted for $80 \%$ of all cervical spine surgeries in that time period, totaling over 1 million procedures. Continuation of this trend was confirmed in a review examining ACDFs from 2007 to 2014, during which the utilization of this procedure increased by another $92 \% .{ }^{13}$ Both of these escalations were marked by significant increases in patient age and number of comorbidities, as well as the costs associated with patient care. With in-hospital costs for an ACDF totaling up to
$\$ 24,000$, a 90 -day readmission rate as high as $7.7 \%$, and one-third of these readmitted patients returning for an infection that can carry an additional $\$ 100,000$ in costs, limiting the need for reoperation in this increasingly elderly and morbid patient cohort is of utmost importance. .,4,27 $^{2}$

Even as the downsides to PEEK use become increasingly clear, the question remains as to what drives its popularity. As discussed in our prior work on single-level ACDF patients, it is possible that billing practices have an impact on graft selection. ${ }^{7}$ CPT codes demonstrate significantly higher reimbursement rates for a PEEK interbody device versus structural allograft, with work relative value units (wRVU) of 4.25 versus 1.81, respectively. This is compounded in the context of multiple-level procedures, as synthetic cage use is billed per level of placement, whereas allograft use is billed just once per operation..$^{21}$ The total wRVU for a standard 3-level plated ACDF with PEEK implants is approximately 64.81, while the same procedure using structural allograft totals 52.21 wRVU. Virk et al. found the cost of even a single-level ACDF with a PEEK cage to be significantly higher than an ACDF with allograft $(\$ 18,314$ vs $\$ 12,539) .{ }^{24}$ This price discrepancy carries broader implications on a systems level given that the United States topped \$3.5 trillion in healthcare utilization costs in 2017..$^{12,18}$

\section{Study Limitations}

The main study limitation is its retrospective nature and the inadvertent biases that this study design may introduce. Bone quality studies were not routinely done, so it is possible that a difference in bone health could have existed between the groups. In terms of the surgical procedure, standardized use of plate and screw fixation would improve the comparison between the 2 groups. We have included the longest possible follow-up time for each patient, though this resulted in a difference between average follow-up times for the 2 groups. This difference was deemed not to be an issue, however, as rates of pseudarthrosis were higher in the PEEK group despite having longer average follow-up time for fusion to occur. Future studies could set a minimum follow-up time of 2 years (instead of 1 year) to most accurately capture fusion rates; however, in this study the average radiographic follow-up was more than 2 years in the allograft group and nearly 3 years in the PEEK group. While CT is considered by many to be the gold standard for radiographic assessment of pseudarthrosis, not every patient had a cervical spine CT performed at least 1 year postsurgery. Flexion and extension radiographs were included in our review, as these are more useful than static radiographs for assessing fusion status. ${ }^{7}$ Finally, there were 13 different surgeons involved in these procedures, which makes standardization of technique and practice patterns difficult but does increase the external validity of our findings. Future prospective studies may provide further evidence on this topic.

\section{Conclusions}

This study reinforces the findings from our institution's prior work on single-level ACDF procedures and similarly demonstrates a greater than 6-fold increase in the rate 
of nonunion and a statistically significantly higher reoperation rate in multiple-level ACDF patients who received PEEK implants compared with those who received structural allograft. Patients who have undergone ACDF procedures of more than 1 level are particularly prone to pseudarthrosis and are more likely to become symptomatic and require reoperation. The costs incurred in these scenarios can number well into the hundreds of thousands of dollars, not to mention the cost of increased duress to the patient. Caution should be taken when determining implant substrate, as this choice offers an immediate and objective way to reduce the risk profile of the patient, a decision that is increasingly important as the population being treated with ACDF surgery becomes older and sicker. Insurers should be encouraged to recognize this discrepancy and adjust reimbursement rates accordingly.

\section{Acknowledgments} tance.

The authors thank Shirley McCartney, $\mathrm{PhD}$, for editorial assis-

\section{References}

1. Chen F, He W, Mahaney K, Noeller J, Mhanna N, Viljoen S, et al: Alternative grafts in anterior cervical fusion. Clin Neurol Neurosurg 115:2049-2055, 2013

2. Daniels AH, Kawaguchi S, Contag AG, Rastegar F, Waagmeester G, Anderson PA, et al: Hospital charges associated with "never events": comparison of anterior cervical discectomy and fusion, posterior lumbar interbody fusion, and lumbar laminectomy to total joint arthroplasty. J Neurosurg Spine 25:165-169, 2016

3. Delloye C, Cornu O, Druez V, Barbier O: Bone allografts: What they can offer and what they cannot. J Bone Joint Surg Br 89:574-579, 2007

4. Goz V, Rane A, Abtahi AM, Lawrence BD, Brodke DS, Spiker WR: Geographic variations in the cost of spine surgery. Spine (Phila Pa 1976) 40:1380-1389, 2015

5. Hee HT, Kundnani V: Rationale for use of polyetheretherketone polymer interbody cage device in cervical spine surgery. Spine J 10:66-69, 2010

6. Kolstad F, Nygaard OP, Andresen H, Leivseth G: Anterior cervical arthrodesis using a "stand alone" cylindrical titanium cage: prospective analysis of radiographic parameters. Spine (Phila Pa 1976) 35:1545-1550, 2010

7. Krause KL, Obayashi JT, Bridges KJ, Raslan AM, Than KD: Fivefold higher rate of pseudarthrosis with polyetheretherketone interbody device than with structural allograft used for 1-level anterior cervical discectomy and fusion. J Neurosurg Spine 30:46-51, 2018

8. Kurtz SM, Devine JN: PEEK biomaterials in trauma, orthopedic, and spinal implants. Biomaterials 28:4845-4869, 2007

9. Lee DH, Cho JH, Hwang CJ, Lee CS, Cho SK, Kim C, et al: What is the fate of pseudarthrosis detected 1 year after anterior cervical discectomy and fusion? Spine (Phila Pa 1976) 43:E23-E28, 2018

10. Lu DC, Tumialán LM, Chou D: Multilevel anterior cervical discectomy and fusion with and without rhBMP-2: a comparison of dysphagia rates and outcomes in 150 patients. J Neurosurg Spine 18:43-49, 2013

11. Maharaj MM, Phan K, Mobbs RJ: Anterior cervical discectomy and fusion (ACDF) autograft versus graft substitutes: what do patients prefer? A clinical study. J Spine Surg 2:105-110, 2016

12. Martin AB, Hartman M, Washington B, Catlin A: National health care spending in 2017: growth slows to post-great recession rates; share of GDP stabilizes. Health Aff (Millwood) 38:101377hlthaff201805085, 2019

13. Martin CT, D’Oro A, Buser Z, Youssef JA, Park JB, Meisel HJ, et al: Trends and costs of anterior cervical discectomy and fusion: a comparison of inpatient and outpatient procedures. Iowa Orthop J 38:167-176, 2018

14. Oglesby M, Fineberg SJ, Patel AA, Pelton MA, Singh K: Epidemiological trends in cervical spine surgery for degenerative diseases between 2002 and 2009. Spine (Phila Pa 1976) 38:1226-1232, 2013

15. Phillips FM, Carlson G, Emery SE, Bohlman HH: Anterior cervical pseudarthrosis. Natural history and treatment. Spine (Phila Pa 1976) 22:1585-1589, 1997

16. Pirkle S, Kaskovich S, Cook D, Ho A, Shi LL, Lee MJ: Cages in ACDF are associated with a higher nonunion rate than allograft: a stratified comparative analysis of 6130 patients. Spine (Phila Pa 1976) 44:384-388, 2019

17. Pollock R, Alcelik I, Bhatia C, Chuter G, Lingutla K, Budithi $\mathrm{C}$, et al: Donor site morbidity following iliac crest bone harvesting for cervical fusion: a comparison between minimally invasive and open techniques. Eur Spine J 17:845-852, 2008

18. Saifi C, Fein AW, Cazzulino A, Lehman RA, Phillips FM, An HS, et al: Trends in resource utilization and rate of cervical disc arthroplasty and anterior cervical discectomy and fusion throughout the United States from 2006 to 2013. Spine J 18:1022-1029, 2018

19. Schnee CL, Freese A, Weil RJ, Marcotte PJ: Analysis of harvest morbidity and radiographic outcome using autograft for anterior cervical fusion. Spine (Phila Pa 1976) 22:22222227, 1997

20. Sethi A, Craig J, Bartol S, Chen W, Jacobson M, Coe C, et al: Radiographic and CT evaluation of recombinant human bone morphogenetic protein-2-assisted spinal interbody fusion.

AJR Am J Roentgenol 197:W128-W133, 2011 (Erratum in AJR Am J Roentgenol 197:1024, 2011)

21. Singh K, Qureshi S: ISASS Policy Statement-cervical interbody. Int J Spine Surg 8:13, 2014

22. Steinhaus ME, Hill PS, Yang J, Feuchtbaum E, Bronheim RS, Prabhakar P, et al: Urinary N-telopeptide can predict pseudarthrosis after anterior cervical decompression and fusion: a prospective study. Spine (Phila Pa 1976) 44:770-776, 2018

23. Vaidya R, Carp J, Sethi A, Bartol S, Craig J, Les CM: Complications of anterior cervical discectomy and fusion using recombinant human bone morphogenetic protein-2. Eur Spine J 16:1257-1265, 2007

24. Virk SS, Elder JB, Sandhu HS, Khan SN: The cost effectiveness of polyetheretheketone (PEEK) cages for anterior cervical discectomy and fusion. J Spinal Disord Tech 28:E482E492, 2015

25. Yoon ST, Konopka JA, Wang JC, Youssef JA, Meisel HJ, Brodke DS, et al: ACDF graft selection by surgeons: survey of AOSpine members. Global Spine J 7:410-416, 2017

26. Yson SC, Sembrano JN, Santos ER: Comparison of allograft and polyetheretherketone (PEEK) cage subsidence rates in anterior cervical discectomy and fusion (ACDF). J Clin Neurosci 38:118-121, 2017

27. Zaki O, Jain N, Yu E, Khan SN: 30- and 90-day unplanned readmission rates, causes, and risk factors after cervical fusion: a single-institution analysis. Spine (Phila Pa 1976) 44:762-769, 2018

\section{Disclosures}

Khoi D. Than is an occasional consultant for Bioventus.

\section{Author Contributions}

Conception and design: Than. Acquisition of data: Teton, Cheaney. Analysis and interpretation of data: Teton, Obayashi. 
Drafting the article: Teton, Cheaney. Critically revising the article: Than, Teton. Reviewed submitted version of manuscript: all authors. Statistical analysis: Obayashi. Administrative/technical/ material support: Than. Study supervision: Than.

\section{Supplemental Information}

\section{Previous Presentations}

Oral presentations of this work were included in the "Top Abstracts" Section of the 35th Annual AANS/CNS Joint Meeting of the Section on Disorders of the Spine and Peripheral Nerves,
Miami, Florida, March 14-17, 2019; the 2019 AANS Annual Scientific Meeting, San Diego, California, April 13-17, 2019; and the 22nd Annual Selby Spine Conference of the Foundation for Orthopaedic Research and Education, Inc., Park City, Utah, January 31 to February 2, 2019.

\section{Correspondence}

Khoi D. Than: Duke University, Durham, NC. khoi.than@duke. edu. 